\title{
Análisis y críticas a las fuentes de financiamiento de los clubes de fútbol profesional chileno
}

\author{
Analysis and criticisms of the sources of financing of Chilean professional football \\ clubs
}

\author{
Mauricio Ríos Lagos ${ }^{1}$ \\ Pontificia Universidad Católica de Valparaíso, Chile
}

\begin{abstract}
RESUMEN El presente artículo tiene el propósito de analizar desde un punto de vista jurídico las fuentes de financiamiento privado con que cuentan los clubes profesionales de fútbol chileno. Se identifica que el carácter de la Organización Deportiva Profesional tendrá importancia para captar recursos, como también la relevancia de los derechos de transmisión en el total de los ingresos de los clubes, identificándose que su reparto genera una desigualdad deportiva que podría limitar el desempeño de algunos de los equipos y, en consecuencia, el acceso a otras fuentes de financiamiento.
\end{abstract}

PAlabras Clave Derecho del Deporte; Derecho del Fútbol; Financiamiento; Organizaciones Deportivas Profesionales; Fondo de Deporte Profesional; Derechos de transmisión.

ABSTRACT The object of the present article is to analyze from a legal point of view the different forms of private financing on which Chilean professional football clubs depend. We identify that the character of the clubs as Professional Sports Organizations is important for attracting resources, and also the contribution of the sale of broadcasting rights to their total income. The distribution of this latter income generates inequalities affecting the sport that could limit the performance of some teams, and consequently their access to other sources of financing.

1. Abogado. Licenciado en Ciencias Jurídicas Pontificia Universidad Católica de Valparaíso, Master of Laws (LL.M) Cornell University, EE.UU. Máster Oficial Derecho, Empresa y Justicia, Universidad de Valencia, España. Profesor Agregado, Pontificia Universidad Católica de Valparaíso. E-mail: mauricio.rios@pucv.cl. (D) https://orcid.org/0000-0003-1571-4279 
KEYWORDS Sports Law; Football Law; Financing; Professional Sports Organizations; Professional Sports Fund; Broadcasting Rights.

\section{Introducción}

En la actualidad el fútbol es una actividad deportiva de alcance mundial, que debido a su profesionalización y popularidad se ha convertido en una actividad lucrativa para los diversos agentes que intervienen en ella, tales como las Federaciones Nacionales, los clubes deportivos, los jugadores, los agentes, el cuerpo técnico y los inversionistas que desean invertir para obtener utilidades.

En el fútbol, al igual que en otras disciplinas, existen los hinchas que están dispuestos a pagar una entrada para ver a su equipo favorito, suscribirse a un cable operador y contratar adicionalmente el canal deportivo respectivo para tener la posibilidad de ver los partidos de ligas nacionales e internacionales, comprar indumentaria deportiva de su equipo o adquirir el denominado merchandising asociado a los mismos.

No debemos olvidar que el fútbol puede analizarse desde distintas perspectivas, la mercantil o comercial, la deportiva o de competición son algunas de ellas. En nuesto caso creemos que no son incompatibles, ya que un equipo exitoso podrá explotar de mejor forma el aspecto comercial y con ello obtener mayores ingresos y a la vez incurrir en gastos al transferir jugadores profesionales, formar a sus propios jugadores e invertir en infraestructura, como lo sería la construcción y mantenimiento de centros deportivos para entrenar e incluso un estadio donde jugar partidos oficiales y amistosos.

La presente investigación pretende analizar los diversos mecanismos de financiamiento privado existentes en el ordenamiento jurídico chileno para los clubes de fútbol profesional así como el gasto y el control de los recursos que se obtienen a través de ellos. Privado en el sentido que dichos recursos no provienen del Estado o de las municipalidades, como podría ser una subvención o ingresos obtenidos a través de un fondo destinado al efecto, sino que por la participación en torneos y competencias organizadas por los organismos del fútbol, así como de la explotación comercial de la actividad deportiva y de las denominadas formas de financiamiento tradicionales a las que puede acceder una empresa.

Se justifica el estudio atendido a que el ordenamiento jurídico chileno presenta una desigualdad en relación al acceso de financiamiento dependiendo de la naturaleza de la organización deportiva que se haya constituido para explotar la actividad, así como también la dependencia que exhiben los clubes deportivos con una fuente de financiamiento en particular, como es el caso de explotación de derechos de transmisión de los partidos o torneos nacionales que se disputan año a año. Sin perjuicio de lo anterior, la normativa nacional, permite a los clubes profesionales de fútbol realizar su actividad como una empresa más que opera en el mercado. Se excluye del presente 
estudio el análisis de las fuentes de ingreso de los jugadores profesionales de fútbol y de la Federación Chilena de Fútbol que deben ser objeto de un análisis particular y diferenciado.

El trabajo comenzará con la descripción del modelo deportivo del fútbol profesional chileno, presentándose un panorama general del mismo; posteriormente se analizará la estructura del fútbol profesional chileno abordando la Sociedad Anónima Deportiva Profesional y la Corporación o Fundación que desarrolla actividades deportivas profesionales; posteriormente se estudiará el financiamiento de los clubes deportivos distinguiendo entre aquellas fuentes propias de la actividad o normativa deportiva y aquellas tradicionales disponibles para los comerciantes y empresas; luego se abordará el gasto y el control de los dineros obtenidos por los clubes deportivos; por último, se presentarán las conclusiones de la investigación.

\section{El Modelo Deportivo del Fútbol Profesional Chileno}

El modelo deportivo del fútbol profesional en Chile está basado en un modelo piramidal de organizaciones deportivas que sigue al europeo en la materia ${ }^{2} \mathrm{y}$ al internacional que deriva del denominado movimiento olímpico, el cual busca integrar el deporte, la cultura, la educación, el juego limpio y los principios éticos universales además de fomentar las relaciones entre los distintos Estados ${ }^{3}$. En la cúspide a nivel nacional nos encontramos con la Federación de Fútbol de Chile (FFCh), la que tiene como socios a la Asociación Nacional de Fútbol Profesional (ANFP) y a la Asociación Nacional de Fútbol Amateur de Chile (ANFA) ${ }^{4}$. En el caso de la ANFP, los clubes deportivos que forman parte de ella son socios o miembros y de acuerdo con el artículo cuarto inciso séptimo de los estatutos, tienen derecho a participar en las competencias que se organicen por la misma, lo que es nuevamente señalado en su artículo quinto, añadiéndose que deben sujetarse a las bases que rijan la respectiva competencia 5 .

El artículo tercero de los estatutos de la ANFP establece el número de cuarenta y dos clubes como máximo de asociados, los cuales participan en la Primera División, Primera B y Segunda División. Lo anterior sin perjuicio que el Consejo de Presidentes (el Consejo) pueda acordar aumentar o reducir el número máximo, como así también aprobar la creación o eliminación de divisiones y la incorporación de nuevos clubes,

2. BLACKSHAW (2017) p. 6.

3. GUERRERO (2013) pp. 126-130.

4. Art.2 de los Estatutos de la Federación de Fútbol de Chile: "Los socios de la Federación son la Asociación Nacional de Fútbol Profesional y la Asociación Nacional de Fútbol Amateur."

5. Art. 5: "Los clubes asociados tendrán derecho a participar en las Competencias que organiza la Asociación Nacional de Fútbol Profesional, sujetándose a las bases que rijan éstas.” 
siendo necesario al efecto un quórum de aprobación de cuatro quintos del total de los votos de los consejeros en ejercicio ${ }^{6}$.

Los objetivos de la ANFP se encuentran contenidos en el artículo primero de sus estatutos, dentro de los cuales debemos destacar la letra b) que señala: "Organizar y promover la calidad de todos los aspectos de los torneos entre los clubes asociados al fútbol profesional." Como es posible observar, la ANFP organiza los torneos y competiciones nacionales en los cuales los clubes profesionales van todos los años a participar, en la búsqueda de logros deportivos y rendimientos económicos.

A nivel internacional, la FFCh forma parte a su vez de la Confederación Sudamericana de Fútbol (CONMEBOL) y en la cúspide del fútbol se encuentra la Féderation Internationale de Football Association (FIFA). La importancia de conocer el funcionamiento del fútbol a nivel nacional e internacional para la presente investigación se encuentra en los torneos y competencia a los cuales los clubes deportivos pueden acceder y a los eventuales premios que puedan recibir por su participación en los mismos, así como también en el cumplimiento de las normativas emanadas de las federaciones nacionales, regionales respectivas y de la FIFA con ocasión de los ingresos que perciban los clubes, como es por ejemplo el dinero por concepto de premios que pueden recibir a medida que avanzan en una competencia internacional.

\section{La Estructura Jurídica del Club de Fútbol Profesional Chileno}

Desde el año 2005, con la dictación de la Ley $\mathrm{N}^{\circ}$ 20.019, 2005, que regula a las Sociedades Anónimas Deportivas Profesionales (Ley SADP) el fútbol chileno pasó, para bien o para mal, a una nueva etapa marcada por un énfasis en la profesionalización del mismo y poniendo en relieve su dimensión comercial. Es posible apreciar en el Mensaje al Ejecutivo del proyecto de Ley que la normativa tenía como propósito “...establecer un modelo de responsabilidad jurídica y financiera para los clubes que desarrollan actividades deportivas de carácter profesional en torno a las cuales se realizan actividades comerciales tales como publicidad, recaudaciones, traspasos de jugadores y venta de derechos por transmisiones televisivas" ${ }^{\prime}$. A lo que se añadía que una de las ventajas de la sociedad que se creaba era la posibilidad de acceder a nuevos recursos ${ }^{8}$.

6. Art. 10: "Son atribuciones y facultades exclusivas del Consejo: "4) Aumentar o reducir el número máximo de clubes establecido en el artículo $3^{\circ}$ que forman la Primera División, Primera B, Segunda División. Aprobar la creación o eliminación de divisiones distintas y elevar o disminuir el número de clubes en cada una de las divisiones. Todo lo anterior deberá ser aprobado por los $4 / 5$ del total de los votos de los Consejeros en ejercicio".

7. Historia de la Ley No 20.019, de 2005, p. 6.

8. Historia de la Ley № 20.019, de 2005, p. 6. 
Lo anterior se condice con el contexto económico que presentaban en la época los distintos clubes deportivos, crisis que los llevaron a algunos de éstos a la quiebra y ser administrados por un Síndico, como es el caso del Club Universidad de Chile y del Club Social y Deportivo Colo-Colo en los años 2006 y 2002 respectivamente. Ambos clubes pasaron luego a ser administrados por sociedades anónimas abiertas a través de un contrato de concesión para los efectos que dichas sociedades saldaran completamente las obligaciones de los clubes y explotaran sus activos9.

Es así como la Ley SADP estable en su artículo primero a la organización deportiva profesional (ODP) como aquellas que tienen "...por objeto organizar, comercializar, producir y participar en espectáculos deportivos..." la que, conforme al artículo cuarto, puede tener el carácter de: (i) Sociedades Anónimas Deportivas Profesionales; y (ii) Corporaciones o Fundaciones. En el último caso, la ley señala expresamente que para desarrollar actividades deportivas profesionales se deben constituir uno o más Fondos de Deporte Profesional (FDP). Además, a las personas jurídicas recién señaladas se debe añadir, como ya se ha dicho, a la sociedad anónima abierta en los casos de Colo-Colo (Blanco y Negro S.A.) y la Universidad de Chile (Azul Azul S.A.).

Los clubes deportivos a través de los años han constituido otras personas jurídicas para ir desarrollando todas sus actividades, entre ellas, sociedades filiales de giro comercial amplio; inmobiliarias; o fundaciones. Así como por ejemplo, podemos mencionar el caso de Colo-Colo que cuenta con la Sociedad Inmobiliaria Estadio Colo-Colo S.A..$^{10}$ o el caso del equipo de Santiago Wanderers y la sociedad anónima abierta Inmobiliaria Santiago Wanderers S.A. ${ }^{11}$

Para efectos de captación de recursos es importante distinguir el tipo de organización deportiva profesional que se constituya, ya que una SADP tiene las características de una sociedad de capital, mientras que una Corporación o Fundación que constituya uno o más FDP no las posee. Recordemos que el proyecto de ley sometido a consideración por el Poder Ejecutivo solo regulaba la constitución de SADP y que durante la tramitación de éste, se estableció la posibilidad de constituir dichos Fondos, pensando más bien en el respeto al derecho de asociación y no en la facilidad o dificultad para la obtención de recursos por parte del club deportivo ${ }^{12}$. Lo anterior puede generar un desequilibrio, en el sentido del acceso al mercado público para obtener financiamiento, ya que de los instrumentos de inversión o de deuda que podrá emitir una SADP, una S.A., tales como acciones o pagarés, solo los últimos estarán disponibles para la Corporación o Fundación que desarrollen actividades deportivas profesionales.

9. Véase al respecto BLANCO Y NEGRO S.A (2019) p. 10 y AZUL AZUL S.A (2019) p. 6.

10. BLANCO Y NEGRO S.A (2019) p. 11.

11. http://inmobiliariasantiagowanderers.cl/?page_id=119.

12. El artículo transitorio del proyecto de ley establecía como obligatorio constituirse como SADP 


\section{El Financiamiento de los Clubes Deportivos de Fútbol Profesional}

En los clubes de fútbol convergen distintos intereses, como la búsqueda de gloria deportiva, los beneficios económicos que puedan derivar de su participación en los torneos y competencias nacionales e internacionales y la explotación comercial de la actividad misma. Hoy los clubes buscan proyectar su rendimiento en un modelo de negocios que les permita captar recursos para continuar siendo competitivos y así mejorar sus resultados en los torneos que disputan cada año.

La captación u obtención de recursos puede realizarse por diversas vías y es lo que hemos de llamar como las fuentes de financiamiento de los clubes de fútbol. Existen distintas formas de financiamiento, partiendo por el origen de los dineros percibidos podemos hablar de fuentes públicas y fuentes privadas, asi existirán dineros provenientes del Estado y/o de organismos descentralizados, como también de inversionistas privados que aportan a través de distintas formas a las arcas de los clubes; hay mecanismos de obtención de recursos propios del fútbol como actividad deportiva, tales como el mecanismo de solidaridad o los premios derivados de la participación en torneos y competencias internacionales y también están las propias de toda empresa que denominamos como tradicionales, como sería la obtención de créditos o la celebración de operaciones mercantiles.

\subsection{Financiamiento propio del fútbol}

Hemos denominado a los ingresos o recursos provenientes de la normativa interna del fútbol como aquellos exclusivos de la misma. Así un club deportivo podrá obtener ingresos por la transferencia de sus jugadores, por la formación y educación de los mismos, el denominado mecanismo de solidaridad y por los premios que pueda obtener por la participación en un torneo o competencia.

\section{a) Los ingresos por la transferencia de jugadores, la formación y educación de los mismos y el mecanismo de solidaridad ${ }^{13}$}

De acuerdo al informe del año 2020 Football Money League, elaborado por Deloitte, algunos de los clubes profesionales de fútbol más importantes del mundo utilizan de forma activa el mecanismo de la transferencia de jugadores como parte su estrategia financiera y que ello puede generarles significativos retornos ${ }^{14}$. En el caso chileno es

para las corporaciones o fundaciones vigentes, lo que posteriormente derivó en ser facultativo e incorporar en la normativa al FDP. Véase al respecto la discusión parlamentaria en Historia de la Ley No 20.019, de 2005, pp. 118 -131.

13. Para un estudio detallado acerca del régimen de transferencia de jugadores véase a CORREA y PINOCHET (2016).

14. DELOITTE SPORTS BUSINESS GROUP (2020) p. 2. 
posible ver efectivamente que los clubes tienen en su política de financiamiento la categoría de ingresos por concepto de transferencia de jugadores ${ }^{15}$. Dicha transferencia puede reunir el carácter de permanente o ser temporal, conocida también como préstamo.

La normativa interna del fútbol profesional regula la materia en el reglamento sobre el estatuto y la transferencia de jugadores de la FIFA (reglamento de transferencia $)^{16}$ que en su artículo primero establece su ámbito de aplicación, en el apartado número uno señala que dicho reglamento es aplicable entre clubes de distintas asociacione $^{17} \mathrm{y}$ en el apartado dos se establece que la transferencia de jugadores entre clubes de una misma asociación está sujeta a un reglamento elaborado por la misma y que debe ser aprobado por la FIFA ${ }^{18}$. En la práctica, la ANFP no ha dictado un reglamento que regule la materia en forma específica, siendo por lo tanto aplicable el reglamento FIFA y algunas normas del reglamento de la ANFP.

El reglamento de transferencia reconoce además dos tipos de indemnizaciones que pueden surgir por la transferencia de un jugador, la primera de ellas es la relativa a la formación y educación y la segunda es la denominada como mecanismo o contribución de solidaridad ${ }^{19}$. Dichas indemnizaciones surgen con ocasión de los denominados derechos de formación de los deportistas que consisten en una "..cantidad económica que tiene por objeto compensar el gasto que ha supuesto para una entidad deportiva la actividad destinada a la preparación de un deportista cuando éste pasa a formar parte de otro equipo..." ${ }^{20}$.

15. Un ejemplo es el caso de la Universidad Católica que reconoce expresamente en su Memoria Anual del año 2019, que Cruzados S.A.D.P considera, en su modelo de negocios, como parte fundamental de su financiamiento la venta de pases de jugadores, véase al respecto CRUZADOS SADP (2019) p. 50.

16. Reglamento sobre el estatuto y la transferencia de jugadores de la FIFA. Edición de Febrero 2021. 17. Art. 1.1: "Este reglamento establece las normas mundiales y obligatorias concernientes al estatuto de los jugadores y su elegibilidad para participar en el fútbol organizado, así como su transferencia entre clubes de distintas asociaciones".

18. Art. 1.2: "La transferencia de jugadores entre clubes de una misma asociación está sujeta a un reglamento específico, redactado por la asociación correspondiente conforme al art. 1, apdo. 3 del presente reglamento, el cual debe ser aprobado por la FIFA. Dicho reglamento establecerá las disposiciones para la resolución de disputas entre clubes y jugadores, de acuerdo con los principios estipulados en el presente reglamento. Asimismo, establecerá un sistema para recompensar a los clubes que invierten en la formación y la educación de jugadores jóvenes."

19. En materia sobre los conflictos que pueden surgir entre los clubes y jugadores en relación a la aplicación del reglamento y los órganos competentes véase a RÍOS y ÁLVAREZ (2019) p. 126.

20. DE LA IGLESIA (2016) p. 145. 
La indemnización por formación se encuentra regulada en el artículo $2 \mathrm{O}^{21} \mathrm{y}$ en el Anexo 4 del reglamento de transferencia, estableciéndose los presupuestos para su pago, la responsabilidad del mismo, los costos de formación y el cálculo de ella, así como las eventuales medidas disciplinarias que puedan aplicarse ante el incumplimiento de pago. La solidaridad está recogida en el artículo $21^{22}$ y el Anexo 5 , regulándose los aspectos relativos a la contribución y el procedimiento de pago de ésta.

En nuestro ordenamiento jurídico nos encontramos con el reconocimiento expreso de la indemnización por formación, siendo aplicable el Código del Trabajo que regula en el Libro I Título II Capítulo VI el contrato de los deportistas profesionales y trabajadores que desempeñan actividades conexas. En efecto, el artículo 152 bis E establece una indemnización por concepto de formación y educación que debe pagar el equipo que contrata a un futbolista que celebra su primer contrato de trabajo en calidad de profesional con un club deportivo distinto al o a los que participaron en ellas. Así la indemnización la recibe o reciben los equipos que formaron al jugador ${ }^{23}$. El Código del Trabajo entrega a la entidad superior de la respectiva disciplina deportiva, en este caso la FIFA, la determinación de los montos por concepto de indemnización por el denominado derecho de formación, siendo aplicable el reglamento de transferencia.

21. Art. 20 Indeminzación por formación. "La indemnización por formación se pagará al club o clubes formadores de un jugador: 1) cuando el jugador se inscriba por primera vez como profesional y 2) por cada transferencia del jugador profesional hasta el fin del año natural en el que cumple 23 años. La obligación de pagar una indemnización por formación surge, aunque la transferencia se efectúe durante o al término del contrato. Las disposiciones sobre la indemnización por formación se establecen en el anexo 4 del presente reglamento. Los principios de la indemnización por formación no serán aplicables al fútbol femenino."

22. Art. 21. Mecanismo de solidaridad. "Si un jugador profesional es transferido antes del vencimiento de su contrato, el club o los clubes que contribuyeron a su educación y formación recibirán una parte de la indemnización pagada al club anterior (contribución de solidaridad). Las disposiciones sobre la contribución de solidaridad se establecen en el anexo 5 del presente reglamento."

23. Artículo 152 bis E.- Cuando un deportista celebre su primer contrato de trabajo en calidad de profesional con una entidad deportiva distinta a la o las participantes en su formación y educación, aquélla deberá pagar a estas últimas una indemnización en razón de la labor formativa realizada, de acuerdo a las normas fijadas por la entidad superior de la respectiva disciplina deportiva.

Dicho pago estará dirigido únicamente a compensar la formación del deportista, y deberá tener en cuenta, al fijar la referida indemnización, la participación proporcional entre las distintas entidades deportivas participantes en la formación y educación de estos deportistas. 
En el Anuario Financiero del Fútbol Chileno, elaborado por la ANFP, se señala que la transferencia de jugadores, derechos de formación y mecanismos de solidaridad representó un 16\% del total de ingresos en el año $2019^{24}$. Considerando solo a la Primera División, el porcentaje es un poco mayor correspondiendo a un $18 \%$ y en el caso de los equipos de Colo-Colo el 24\%, la Universidad Católica el 19\% y a la Universidad de Chile solo el $7 \%^{25}$.

\section{b) Los ingresos por la partipación en torneos y competencias}

Lo propio del fútbol y del deporte profesional es la competencia de los equipos en torneos que se celebren año a año en el cual se busque a un campeón que se lleve la gloria deportiva máxima y que en algunos casos igualmente reportará beneficios económicos no solo al equipo que haya levantado el trofeo, sino que al resto de ellos que tuvo la oportunidad de participar en el campeonato. Los ingresos que se obtengan por la participación en determinados torneos permiten el cumplimiento de fines deportivos y económicos para el club de fútbol.

En América del Sur tenemos las copas Libertadores y Sudamericana organizadas cada año por la CONMEBOL y que constituyen un claro ejemplo de la relación entre los fines deportivos y económicos. Así, a nivel nacional la ANFP en las bases de competencia de los torneos locales regula la participación por clasificación de los equipos chilenos a las competencias internacionales ${ }^{26}$. Lo anterior es un claro incentivo para los clubes deportivos ya que una buena campaña puede llevarlos a disputar los torneos en busca del éxito deportivo colectivo, sin perjuicio del éxito personal de los jugadores que esperan que una actuación destacada puede resultar también en reconocimientos deportivos y económicos, como lo sería una transferencia a un club sudamericano o europeo y la celebración de contratos de patrocinio.

Cada año la CONMEBOL anuncia los premios económicos que recibirán los equipos que participen en las competencias y si se toma el ejemplo de la Copa Libertadores, la confederación señala que ellos “...provienen del apoyo constante que brindan a la CONMEBOL Libertadores, sus aliados comerciales y titulares de los derechos audiovisuales. Varias empresas de importancia global han invertido en los derechos que han adquirido para asociar sus marcas de forma exclusiva con la Conmebol y la Conmebol Libertadores" ${ }^{27}$. Para la edición 2020 de la Copa Libertadores el campeón del

24. ASOCIACIÓN NACIONAL DE FÚTBOL PROFESIONAL (2020) p. 10.

25. ASOCIACIÓN NACIONAL DE FÚTBOL PROFESIONAL (2020) pp. 29-31.

26. Por ejemplo el Título XI "De la Clasificación a torneos internacionales", artículo 85 y ss de las Bases del Campeonato Nacional de Primera División, Temporada 2021 regula la Clasificación de clubes chilenos a la Copa CONMEBOL Libertadores 2022 y la Copa CONMEBOL Sudamericana 2022.

27. Manual de Clubes CONMEBOL Libertadores 2021, p. 20. 
torneo recibió en premios una suma total de USD 22.550.00o, correspondiento dicho monto al dinero obtenido por avanzar las diferentes fases del campeonato sumado a la victoria en la final que ascendió a la suma de USD 15.00o.0oo del total señalado ${ }^{28}$.

Es posible constatar que la participación en las competencias internacionales pueden generar ingresos por premios económicos a los clubes deportivos, dineros que irán aumentando de forma progresiva a medida que vayan avanzando las distintas etapas de los torneos. A lo anterior hay que agregar dineros que se obtengan por las ventas de entradas por los partidos de locales y los derechos de transmisión que puedan corresponderle. Si tomamos por ejemplo el caso de la Universidad Católica, su participación a nivel internacional en el año 2019 le reportó ingresos de transmisión en la Copa Libertadores por \$2.023 millones de pesos y por la Conmebol Sudamericana $\$ 264$ millones de pesos ${ }^{29}$.

\section{c) Ingresos por la explotación de los derechos de transmisión y reproducción}

El modelo de televisión pagada aplicado al fútbol ${ }^{30}$, es el utilizado por la ANFP y que tiene su origen en el año 2003, siendo uno de los principales soportes financieros de los clubes de fútbol ${ }^{31}$, encontrándose en tela de juicio su continuidad producto del requerimiento presentado por la Fiscalía Nacional Económica (FNE) en diciembre del año 2020 contra el Canal del Fútbol SpA (CDF), hoy TNT Sports ${ }^{32}$. Además, debemos tener presente que los dineros percibidos por la explotación de los derechos de transmisión constituyen a su vez el principal mecanismo de financiamiento a nivel mundial ${ }^{33}$.

28. https://www.conmebol.com/es/el-camino-de-los-premios-y-millones-del-campeon-de-la-conmebol-libertadores.

29. CRUZADOS SADP (2019) p. 32.

30. En España, que cuenta con una de las ligas más importantes del mundo, se han reconocido dos sistemas o modelos para la venta de derecho audiovisuales de los clubes, la venta individualizada que puede realizar cada club o una venta centralizada que se efectúa en su conjunto. Véase un estudio al respecto en CAÑIZARES (2015) pp. 27-44.

31. Los ingresos por derechos de transmisión para la Primera B corresponden al $67 \%$ del total de sus ganancias durante el año 2019, considerablemente mayor que la Primera División, que corresponde a un 39\% lo que complementa con ingresos provenientes de patrocinios, publicidad o merchandising, entre otros; de transferencias de jugadores; de ingresos por la venta de tickets y abonados y de otros ingresos como premios y/o aportes. En detalle encontramos estos antecedentes en el Anuario Financiero del Fútbol Chileno del año 2020.

32. Desde el 17 de enero de 2021 denominado TNT Sports. Requerimiento Rol C-411-2020. Disponible en http://consultas.tdlc.cl/do_search?proc=3\&idCausa $=42231$.

33. DELOITTE SPORTS BUSINESS GROUP (2020) pp. 4-6. 
El año 2003 es clave para comenzar a apreciar la transformación que sufre el fútbol chileno, transformación en el sentido de visualizar grandes oportunidades de comercialización de este deporte, puesto que nace el $\mathrm{CDF}^{34}$, sociedad de responsabilidad limitada controlada por la ANFP con un $80 \%$ de participación y el $20 \%$ restante por la sociedad Gestión de Televisión Ltda.(GDT) ${ }^{35}$. Hoy esto se ha modificado puesto que en el año 2018 la FNE aprobó la operación de concentración de dos empresas del área de las telecomunicaciones en Chile: Turner International Latin America, Inc. (TILA) ${ }^{36}$ y Servicios de Televisión Canal del Fútbol Limitada (CDF). La forma de realizar esta operación de concentración fue adquiriendo la ANFP la participación de GDT en la sociedad, para luego transferir la totalidad de las acciones de CDF a TILA, transformando con anterioridad al CDF en una sociedad por acciones ${ }^{37}$. Luego, la ANFP y el CDF suscribieron un contrato de Licencia, por el que el CDF tuviera durante 15 años los derechos exclusivos de transmisión televisiva del Campeonato Nacional, la Copa Chile y la Super $\mathrm{Copa}^{38}$.

El artículo primero letra $\mathrm{m}$ ) de los Estatutos de la ANFP, señala que uno de los objetivos de la asociación es: "Celebrar todos los actos y contratos que tengan por objeto ceder, comercializar, distribuir o licenciar los derechos de transmisión y reproducción por cualquier medio de los campeonatos, torneos, eventos y partidos organizados por la Asociación, pudiendo negociar y acordar en ellos todos sus términos y condiciones"; por otra parte la letra n) del mismo artículo señala que otro de los objetivos es: "Ejercer todos y cada uno de los derechos que a la Asociación le correspondan conforme a los términos y condiciones de los contratos referidos en la letra m) precedente" 39 .

\footnotetext{
34. Sociedad dedicada a la gestión y producción del canal CDF en sus distintas señales. Opera en los segmentos de producción de programas y contenidos; provisión de programas y canales mayoristas para terceros operadores, venta de programación original, comercialización de contenidos no lineales y venta de espacios de publicidad.

35. Sociedad encargada de mantener y administrar la inversión realizada en CDF. Resolución de aprobación de la operación de concentración de la FNE de fecha 14 de diciembre de 2018, Rol F-1162018. Disponible en https://www.fne.gob.cl/wp-content/uploads/2018/12/aprob54a_F116_2018. pdf.

36. TILA es una empresa constituida bajo las leyes del Estado de Georgia, Estados Unidos, que tiene como objeto el licenciamiento mayorista de canales de televisión de pago y que agrupa a los canales de televisión latinoamericano de Turner Broadcasting System (Turner). Disponible en https://www. fne.gob.cl/wp-content/uploads/2018/12/inap_F116_2018.pdf.
}

37. Organización jurídica necesaria, ya que la ANFP al adquirir todos los derechos de GDT reúne el $100 \%$ de participación en la sociedad por lo que se disuelve o se transforma en una persona jurídica unipersonal, esto es una sociedad por acciones.

38. ASOCIACIÓN NACIONAL DE FÚTBOL PROFESIONAL (2020) p. 15.

39. Estatutos de la ANFP 2020. 
En el mismo sentido el artículo 19 letra v), donde se establecen las facultades y deberes del Directorio, prescribe que es éste organismo el que debe "Celebrar previa autorización del Consejo de Presidentes, todos los contratos para transmitir por cualquier medio audiovisual de transmisión, los partidos de las competencias que organice la Asociación.... Es así como el Directorio es quien debe celebrar los convenios y suscribir los contratos que tengan como fin la transmisión de los partidos, sea a través de la televisión abierta, cerrada, por cable aéreo o internet, entre otros. Desde el año 2013 existe la posibilidad de que los partidos de la Primera División y de la Primera B sean transmitidos vía Streaming a través de la aplicación Estadio TNT Sports (anteriormente Estadio CDF), para lo que se debe tener una cuenta en TNT Sports y pagar mensualmente la suma de USD 17.

En caso que los partidos de la competencia de una División no se encuentren contemplados en los contratos sucritos, el Directorio con el acuerdo escrito de a lo menos la mayoría de los equipos integrantes de la respectiva División, puede celebrar los convenios y contratos de transmisión de televisión. Sin embargo, mediante la televisión abierta, no se pueden transmitir dichos partidos a la misma ciudad ni en un radio de $100 \mathrm{kms}$. a la redonda donde se disputen. Sin embargo, el club que juega de local podría consentir expresamente en ello y solamente en los casos en que no se juegue otro partido de la competencia el mismo día ${ }^{40}$.

Tratándose de transmisión televisiva de partidos amistosos internacionales, el Directorio puede autorizar, si lo solicita el club chileno, dichas transmisiones y siempre que ese día no se juegue un partido de la competencia nacional o de las Selecciones Nacionales además de que deben mediar a lo menos dos días entre los partidos y el anterior y el próximo ${ }^{41}$. Del mismo modo, la Asociación tiene el derecho exclusivo y excluyente de comercializar con las distintas empresas de televisión u otras similares, la exhibición de los goles de las competencias que organice, además de los compactos de los partidos y los programas de televisión que exhiben los goles y compactos, lo que se condice con el actual modelo utilizado, ya que predomina el derecho de imagen, activos que se transan mediante los distintos contratos de exclusividad ${ }^{42}$.

40. Artículo 19 letra v) inciso segundo de los Estatutos de la ANFP.

41. Artículo 19 letra v) inciso tercero de los Estatutos de la ANFP.

42. Si recordamos décadas atrás, los compactos y goles se transmitían cada domingo en programas dedicados al deporte, abarcando gran parte de éstos, lo relacionado con los partidos de futbol de cada semana, un ejemplo es Zoom Deportivo. Artículo 19 letra v) inciso cuarto de los Estatutos de la ANFP. 
El artículo 19 letra v) inciso sexto debe complementarse con el artículo 55 de los estatutos, ya que se otorga un mandato gratuito e irrevocable por parte de los clubes a la ANFP para actuar en su nombre y representación en todos los actos y contratos relativos a la oferta, venta, comercialización, uso, goce, explotación, arrendamiento, licencia u otro, respecto a los derechos de transmisión televisiva relativa a los partidos y otros espectáculos deportivos relacionados con torneos y competencias deportivas. En este sentido se contempla este mandato sin perjuicio de que la ANFP haya cedido, comercializado o licenciado los derechos a un tercero.

Los ingresos obtenidos son distribuidos por la ANFP entre los diferentes clubes mediante la decisión de su Consejo de Presidentes, lo que se encuentra regulado en el artículo 10 número 13 de los estatutos, señalando que el Consejo debe determinar y acordar la forma de distribución de los pagos que recibirán los clubes con motivo de los contratos celebrador por la ANFP que digan relación con la cesión, comercialización, distribución o licencia de los derechos de transmisión televisiva de las competencias en que participan los clubes de Primera División y Primera B.

Es así como el pago de los ingresos provenientes del contrato de licencia de los derechos de transmisión televisiva o de ingresos provenientes de la explotación, aprovechamiento, venta o comercialización de dichos derechos, se realiza anualmente en 12 cuotas mensuales de la siguiente forma ${ }^{43}$ :

i. Si el monto total a repartir entre los clubes, es igual o inferior a UF 1.441.369,94, éste se reparte de acuerdo a los siguientes porcentajes: i) el $25 \%$ entre los clubes Colo-Colo, Universidad de Chile y Universidad Católica, según la forma de distribución que ellos informen; ii) el 49,4\% entre los restantes clubes que al momento de percibir dichos ingresos participen de la Primera División, por partes iguales; y iii) el 25,6\% entre los clubes que al momento de percibir los ingresos participen de la Primera B, por partes iguales.

ii. Si el monto total a repartir entre los clubes es mayor a las UF 1.441.369,44, el monto en exceso de tal cantidad se reparte de la siguiente forma: i) el 30\% entre los clubes que al momento de percibir los ingresos participen de la Primera B, por partes iguales; y, ii) el 70\% será distribuido entre los clubes que al momento de percibir los ingresos participen de la Primera División.

43. Artículo $10 \mathrm{n}^{\circ} 13$ de los Estatutos de la ANFP. 
Sumado a lo anterior se ha estipulado que sin perjuicio de lo recién señalado, los clubes Colo-Colo, Universidad de Chile y Universidad Católica, no podrán recibir más del 23\% el año 2017; 20\% el año 2018; 20\% el año 2019; 19\% el año 2020; 18\% el año 2021; $17 \%$ el año 2022; y, 16,56\% desde el año 2023 en adelante hasta el año 2033 o el término del campeonato, lo que resulte más tarde. De acuerdo a los Estados Financieros de la ANFP al 31 de diciembre de 2019-2018, el CDF le entregó a la Asociación la suma total del M\$59.290.085 (miles de pesos) en el año 2019 y M\$47.221.202 (miles de pesos) en el 2018, los que fueron distribuidos a los clubes de acuerdo al mecanismo precedentemente descrito ${ }^{44}$.

La distribución de los dineros recibidos de la forma recién descrita genera un beneficio mayor para los tres clubes más grandes del país de la fuente de financiamiento más importante que tiene en la actualidad el fútbol profesional chileno, lo que puede justificarse por la popularidad de los mismos, lo que lleva a concluir que los partidos de dichos equipos son los más vistos y por ello deban recibir más recursos. Lo anterior, no está exento de críticas ya que el resto de los equipos disponen de menos recursos para invertir en un mejor plantel o infraestructura deportiva lo que desde el punto de vista deportivo no logra cumplirse con algo tan esencial como la igualdad en la competición y a la vez limita o dificulta el acceso a otras fuentes de financiamiento propias del fútbol como lo es participar en torneos internacionales.

\section{d) La explotación de los derechos sobre marcas comerciales}

El título II de la Ley $\mathrm{N}^{\circ} 19.039^{45}$ de 1991, sobre propiedad industrial (LPI) regula las marcas comerciales ${ }^{46}$ cuya explotación constituye una fuente de financiamiento para los clubes de fútbol profesional. Se ha reconocido a la marca comercial las funciones indicadoras de la procedencia empresarial, de calidad, de buena reputación o goodwill

\section{ASOCIACIÓN NACIONAL DE FÚTBOL PROFESIONAL (2020) p. 30.}

45. A partir del año 2006 la encontramos en el DFL 3 que Fija Texto Refundido, Coordinado y Sistematizado de la Ley de Propiedad Industrial.

46. Artículo 19: "Bajo la denominación de marca comercial, se comprende todo signo que sea susceptible de representación gráfica capaz de distinguir en el mercado productos, servicios o establecimientos industriales o comerciales. Tales signos podrán consistir en palabras, incluidos los nombres de personas, letras, números, elementos figurativos tales como imágenes, gráficos, símbolos, combinaciones de colores, sonidos, así como también, cualquier combinación de estos signos. Cuando los signos no sean intrínsecamente distintivos, podrá concederse el registro si han adquirido distintividad por medio del uso en el mercado nacional.

Podrán también inscribirse las frases de propaganda o publicitarias, siempre que vayan unidas o adscritas a una marca registrada del producto, servicio o establecimiento comercial o industrial para el cual se vayan a utilizar.

La naturaleza del producto o servicio al que la marca ha de aplicarse no será en ningún caso obstáculo para el registro de la marca". 
y la publicitaria ${ }^{47}$ que al igual que en otras actividades económicas, cobran cada día más importancia, lo que les permite obtener mayores ventas no solo relacionadas con los éxitos deportivos en un año determinado, sino con el grupo de hinchas que está dispuesto a adquirir los productos o servicios relacionados con la marca.

De acuerdo a la ley, el titular de la marca goza del derecho a utilizarla de forma exclusiva y excluyente en la forma conferida y de distinguir los productos, servicios, establecimientos comerciales o industriales comprendidos en el registro de ella ${ }^{48}$, lo que se conoce como el derecho exclusivo sobre la marca o el aspecto positivo de la misma, que es caracterizado como consustancial y que se relaciona con la función de indicar el origen de los productos o servicios ${ }^{49}$. Aquí podemos ver la posibilidad de explotar comercialmente la marca comercial, a lo que la ley añade el derecho del titular de impedir que un tercero, sin su consentimiento, utilice la marca comercial registrada o marcas similares o idénticas pudiendo inducir a error o confusión entre ellas, también conocido como el aspecto negativo ${ }^{50}$.

Dentro de las formas de explotación de la marca, podemos señalar que la primera de ellas es la que puede realizar el mismo titular, pero a su vez es posible que dicha explotación sea realizada por terceros mediante la celebración de actos y contratos que lo autoricen. Para los clubes deportivos, la posibilidad de que sean terceros quienes lo hagan tiene su fuente normativa en el artículo 14 de la LPI, este último señala que: "Los derechos de propiedad industrial son transmisibles por causa de muerte y podrán ser objeto de toda clase de actos jurídicos, los que deberán constar a lo menos por instrumento privado y se anotarán en extracto al margen del respectivo registro". Así es posible ceder o transferir la marca, o celebrar un contrato de licencia siendo la segunda una posibilidad de financiamiento permanente para ellos. Siguiendo a FERNÁNDEZ-NÓVOA, "Para el licenciante de la marca es esencial la organización empresarial de que dispone el licenciatario a fin de fabricar y distribuir en el mercado los productos diferenciados por la marca licenciada" ${ }^{51}$. Además, como la Ley permite la celebración de toda clase de actos, sería perfectamente posible dar por ejemplo en garantía la marca para obtener financiamiento ${ }^{52}$.

47. Sobre las funciones de las marcas comerciales véase a SCHMITZ (2012).

48. Art. 19 bis D de la Ley No 19.039, de 1991.

49. FÉRNANDEZ-NÓVOA et al. (2017) pp. 581-582.

50. FÉRNANDEZ-NÓVOA et al. (2017) pp. 558-580.

51. FÉRNANDEZ-NÓVOA et al. (2017) p. 619.

52. Con ocasión del estudio de trademarks and passing off del common law, véase BENTLY et al. (2018) p.1172, los autores analizan la posibilidad como mortgages de marcas registradas para obtener financiamento. 
Del contrato de licencia, los clubes deportivos como titulares de una marca comercial y parte licenciante pueden entregar una o más facultades o derechos de que goza el licenciatario por una retribución, como por ejemplo, incorporar la marca en los productos elaborados, poner en el mercado el producto con la marca licenciada y utilizarla como publicidad para difundir los productos que la llevan. Asimismo, es posible que el contrato de licencia tenga o no el carácter de exclusivo, así como las demás condiciones que las partes pueden libremente acordar.

De los clubes deportivos estudiados, podemos mencionar, a modo de ejemplo, algunas marcas comerciales que se encuentran actualmente registradas ante el Instituto Nacional de Propiedad Industrial (INAPI): Santiago Wanderers ${ }^{53}$; Audax Italiano ${ }^{54}$; Unión Española ${ }^{55}$; Cobreloa ${ }^{56}$; y Everton ${ }^{57}$.

\section{e) El patrocinio como forma de financiamiento ${ }^{58}$}

El fútbol por su importancia y popularidad es una oportunidad para que las empresas se relacionen con la FIFA, las federaciones continentales, los clubes deportivos y con los jugadores. Ser patrocinador o sponsor de un evento deportivo como la Copa del Mundo, la Copa América, la Copa Libertadores o tener la oportunidad de que un equipo utilice la ropa deportiva confeccionada por la empresa son solo algunos de los aspectos que hace atractiva la generación de acuerdos comerciales entre ellos. El equipo o la federación respectiva recibirá una prestación a cambio de permitir a la empresa, por ejemplo, incluir su nombre o marca en un evento o en la camiseta del club.

El acuerdo comercial que permite la relación antes indicada es el denominado contrato de patrocinio deportivo mediante el cual el patrocinador se obliga a pagar una suma de dinero o la entrega de productos o servicios al patrocinado, el cual se obliga en cambio a publicitar al primero en los términos y condiciones establecidas por las partes ${ }^{59}$.

53. Número de registro 829705 .

54. Número de registro 835167.

55. Número de registro 1102201.

56. Número de registro 1323653.

57. Número de registro 1289715.

58. Se utilizará la palabra patrocinio desde un punto de vista general para incluir el término auspicio que se ha designado como un contrato con los clubes deportivos, mientras el primero es posible encontrar su referencia exclusiva a la relación contractual con el jugador profesional de fútbol.

59. El contrato de patrocinio deportivo tendría como características: Atipicidad; carácter sinalagmático; consensual; oneroso; no aleatoriedad; intuito personae. Para un análisis del contrato en la legislación española véase a ECHEVERRY (2015) pp. 53-88. 
En relación a las partes del contrato, encontramos al patrocinador y al patrocinado, pudiendo ser tanto personas jurídicas y naturales las que lo celebran ${ }^{60}$, estableciéndose, por ejemplo, un patrocinio a un club deportivo o a un jugador, y de acuerdo a lo que señala BLACKSHAW, el contrato puede tener diversos objetos como es patrocinar un evento deportivo; asignar el nombre del patrocinador a un recinto deportivo; la transmisión de un evento deportivo; patrocinar a un club deportivo o a sus jugadores; obtener designaciones oficiales, como sería acordar ser la aerolínea oficial de un evento, o la tarjeta de crédito oficial; merchandising de productos; hospitalidad corporativa; etc ${ }^{61}$.

En lo que nos interesa, el patrocinio deportivo a los clubes de fútbol puede ser una importante fuente de financiamiento. Así los equipos están en condiciones de negociar distintos tipos de contratos de patrocinio dependiendo de sus necesidades, siendo los principales los celebrados con empresas deportivas para la utilización de sus indumentarias en los campeonatos o torneos que disputen; incorporar el logo o marca de la empresa en la camiseta del equipo, pudiendo ser por ejemplo un empresa bancaria, de telecomunicaciones o dedicada a la elaboración de alimentos; utilizar una bebida isotónica como la bebida oficial del club; etc.

Cada club de fútbol negociará las condiciones de contratación con cada empresa que desee ser patrocinador y así es posible que surjan las denominadas categorías de patrocinadores o sponsors que tendrán una mayor o menor notoriedad o vinculación con el equipo. Un ejemplo de esto constituye el caso de Santiago Wanderers que en su Memoria del año 2019 señala que tiene auspiciadores principales, categoría honor y categoría valor ${ }^{62}$, pero la importancia para la empresa de estar incluido en un lugar determinado de la camiseta queda claramente expresado en lo expuesto por el club: "Para potenciar los ingresos por concepto de auspicios, la gestión realizada fue la de lograr mantener a los auspiciadores por un año más, aún estando en primera B. Y no sólo se pudo mantener a los auspiciadores, sino que además se logró que el último involucrado mostrara mucho más interés en el club, haciendo que ofertara por estar en la espalda superior, desplazando a PF del lugar. No obstante, PF no dejó de invertir en el club, y se mantuvo con auspicios dentro del estadio"63.

Una alternativa de financiamiento originaria de los Estados Unidos es celebrar un acuerdo de denominación del recinto deportivo, conocido como naming rights agreements ${ }^{64}$, por la cual una empresa tiene el derecho de nombrar al recinto deportivo o

60. ECHEVERRY (2015) p. 89.

61. BLACKSHAW (2012) pp. 124-129.

62. CLUB DE DEPORTES SANTIAGO WANDERERS S.A.D.P (2019) p. 43.

63. CLUB DE DEPORTES SANTIAGO WANDERERS S.A.D.P (2019) p. 43.

64. Véase sobre la materia a BLACKSHAW (2012) pp. 165-207. 
arena con fines publicitarios pagando una suma de dinero al o los equipos propietarios de la infraestructura. Un ejemplo de ello es el contrato celebrado el año 2003 entre Toyota, el equipo de básquetbol de la National Basketball Association (NBA) Houston Rockets, el entonces equipo femenino de básquetbol de la Women National Bastketball Association (WNBA) y la empresa Clutch City Sports \& Entertainment, L.P, por el cual la empresa fabricante de automóviles adquirió los namings rights del recinto conocido como Toyota Center ${ }^{65}$.

\section{f) La venta de entradas o abonos para partidos amistosos o disputados en cam- peonatos nacionales e internacionales}

Los partidos disputados en torneos nacionales o internacionales son otra fuente de ingresos o financiamiento de los clubes deportivos. Existe la posibilidad de comprar entradas para cada partido que se dispute o comprar el denominado abono que posibilita la entrada a todos los partidos de local del equipo por un período de tiempo determinado a un precio inferior de la suma individual de cada entrada unido a beneficios adicionales por tener la calidad de abonado. Por ejemplo, en el caso de Universidad Católica, el abono permitía la entrada a los partidos de local en el Campeonato ANFP Plan vital 2020, Copa Chile MTS 2020, partidos CONMEBOL Libertadores 2020 y CONMEBOL Sudamericana 2020, excluyendo los partidos amistosos que el equipo pudiera disputar, así como también no garantizando un número determinado de partidos ya que ello depende del rendimiento del club deportivo ${ }^{66}$.

El abono permite el ingreso de recursos de forma inmediata y no parcializada con el incierto de si efectivamente ellos van a percibirlos o no, ya que dependerá en muchas ocasiones del desempeño del equipo ya que podría estimarse que la compra de entradas puede disminuir o aumentar si el club lleva o no una campaña exitosa en los torneos que disputa. Así, la compra de abonos permite el ingreso a todo evento, independiente de si efectivamente el comprador asiste al estadio o si el equipo tiene mejores o peores resultados.

La figura de la venta de entradas y abono tiene desde el punto de vista jurídico su materialización en la celebración de contratos entre el club deportivo y el abonado, así como también entre el primero y terceros que ofrecerán sus productos o servicios al último, con un descuento previamente acordado bajo el requisito de acreditar la calidad de abonado. Algunos equipos celebran convenios con otras empresas, como es el caso de Punto Ticket S.A., y Ticketplus SpA para la compra de entrada o abonos on-line para con ello facilitar la compra ${ }^{67}$.

65. https://www.nba.com/rockets/news/toyota_center_030724.html.

66. https://tienda.cruzados.cl/pages/terms-and-conditions.

67. Los equipos Universidad de Chile y Unión Española permiten abonarse a través del sitio web 
Hasta el momento hemos hablado de la persona que adquiere una entrada o el abono, pero también debemos referirnos a la categoría conocida como socio de un club deportivo de fútbol. Algunos clubes distinguen entre socio y abonado, mientras otros mantienen solo el término de socio para la captación de ingresos. La calidad de socio puede tener diversas manifestaciones dependiendo del equipo: El socio paga una suma anual para ver los partidos de local y accede a diversos beneficios otorgados por el club o por terceros con los cuales el equipo celebró el convenio respectivo ${ }^{68}$; el socio tiene descuentos en la compra de entradas, como asimismo participación en la asamblea de socios y el acceso a descuentos que realiza el club o terceros sobre productos y servicios ${ }^{69}$.

En la primera figura del socio, se muestra una similitud con el abonado mientras que en la segunda, el socio puede obtener descuentos en entradas, ofrecimiento de preventa exclusiva y lo que para un hincha del club respectivo puede ser muy atractivo es la participación en la asamblea de socios o del club y generar así la posibilidad de que la persona en su calidad de socio tenga voz y/o voto en las mismas ${ }^{70}$.

Las bases de los campeonatos regulan la venta de entradas y la distribución de lo recaudado por este concepto y en caso del campeonato nacional de primera división del año 2021, se dispone en el título XI "Del valor de las entradas, distribución, canje y manejo de la recaudación", que el titular de la totalidad de los montos recaudados es el Club deportivo que actúe como local, teniendo a su vez que emitir a la ANFP una copia de la liquidación donde conste la cantidad de público que asistió como la cantidad o el monto de lo recaudado ${ }^{71}$.

La importancia de la venta de entradas, en alguna de las formas ya señaladas, en los ingresos de los clubes de fútbol asciende al $12 \%$ del total ${ }^{72}$, correspondiendo en la temporada 2019 a Colo-Colo, Universidad de Chile y Universidad Católica ser los equipos con mayor venta o ticketing ${ }^{73}$ y teniendo en consideración que la temporada

de Punto Ticket S.A., mientras que la Universidad Católica y Santiago Wanderers por la página de Ticketplus SpA.

68. Tal sería el caso de Santiago Wanderers en el cual el socio del club tiene las características propias de un socio y además de un abonado al pagar la suma por las entradas https://www.santiagowanderers.cl/seccion/78/hazte-socio.html.

69. Tomando el ejemplo de la Universidad Católica, se distingue al socio cruzado y al socio abonado, siendo el primero el que participa de la calidad tradicional de socio presentada en el presente trabajo, mientras que el segundo es el considerado como su propio nombre lo señala como abonado.

70. Por ejemplo, Colo-Colo y Universidad Católica señalan que la calidad de socio permite la participación en la Asamblea de Socios respectiva. Véase al respecto en el caso de la UC https://tienda. cruzados.cl/events/SocioCruzado y en el de Colo-Colo https://mi.csdcolocolo.cl/faqs/que-beneficios-tengo-por-hacerme-sociasocio.

71. Art. 65 de las Bases Campeonato Nacional de Primera División Temporada 2021.

72. ASOCIACIÓN NACIONAL DE FÚTBOL PROFESIONAL (2020) p. 10.

73. ASOCIACIÓN NACIONAL DE FÚTBOL PROFESIONAL (2020) p. 27. 
fue afectada por el denominado estadillo social de octubre de 2019 que obligó a la ANFP a suspender por razones de fuerza mayor el fútbol profesional chileno en sus distintas categorías ${ }^{74}$. Esta fuente de financiamiento se ha visto igualmente disminuida por el COVID-19 que no permite la celebración de partidos con público.

\subsection{El financimiento tradicional de los clubes deportivos}

Por último corresponde enunciar las denominadas formas tradicionales de financiamiento que un club de fútbol dispone en su dimensión comercial tradicional, ya que se encuentran disponibles para las empresas en general, independiente de la actividad que puedan desarrollar: a) La obtención de créditos ante los bancos e instituciones financieras; b) la emisión de títulos valores de inversión o deuda; c) la celebración de operaciones comerciales como sería por ejemplo el caso del leasing; o, d) el arrendamiento de infraestructura a terceros para la realización de eventos.

Un aspecto importante que destacar es la estructura u organización jurídica que pueda tener el club de fútbol, ya que en el caso chileno las SAA Blanco y Negro S.A. y Azul Azul S.A se encuentran inscritas en el registro de valores que lleva la Comisión para el Mercado Financiero (CMF) y pueden emitir valores de oferta pública para financiar sus proyectos bajo la normativa de la Ley $N^{\circ} 18.045$, de 1981, de Mercado de Valores (LMV) y las normas de carácter general que la CMF dicta al respecto.

En el caso de las SADP, que es un tipo de sociedad anónima, permite la aplicación del artículo quinto de la LMV pudiendo, por ejemplo, voluntariamente inscribir en el registro de valores las acciones emitidas, y también ser un emisor de valores de oferta pública, en cuyo caso será obligatoria la inscripción ${ }^{75}$, pudiendo emitir alguno de los valores que señala el artículo tercero de la LMV, siendo títulos de inversión o de deu-

74. https://www.anfp.cl/noticia/34674/comunicado-oficial.

75. Art. 5: "La Superintendencia llevará un Registro de Valores el cual estará a disposición del público.

En el Registro de Valores se deberán inscribir:

a) Los emisores de valores de oferta pública;

b) Los valores que sean objeto de oferta pública;

c) Las acciones de las sociedades anónimas que tengan 500 o más accionistas o, a lo menos, el 10\% de su capital suscrito pertenezca a un mínimo de 100 accionistas, excluidos los que individualmente o a través de otras personas naturales o jurídicas, excedan dicho porcentaje, y

d) Las acciones emitidas por sociedades anónimas que voluntariamente así lo soliciten o que por obligación legal deban registrarlas.

La solicitud de inscripción de un emisor en el registro de valores deberá estar necesariamente acompañada de una solicitud de inscripción de los valores que dicho emisor ofrecerá públicamente. Sin embargo, no estarán obligados a ofrecer los valores inscritos sino hasta después que transcurra un año desde su registro. 
da tales como lo serían las acciones, pagarés, letras o bonos ${ }^{76}$. Es interesante destacar que la SADP puede no estar obligada o decidir voluntariamente no registrar sus valores y no acceder al Mercado de Valores para buscar financiamiento en un mercado que es público, también es posible que ella decida hacerlo mediante la emisión de valores de oferta privada no sometido a la normativa de la LMV ni a la normativa y fiscalización de la CMF en la materia.

En el caso de la Corporación o Fundación que haya constituido un FDP la regulación no parece ser clara para dar una respuesta, así se le permite acceder al mercado de valores para buscar financiamiento. Lo anterior es debido a que surge la pregunta de si es posible o no que un FDP pueda ser un emisor de valores de oferta pública, lo que si es posible es que la persona jurídica de derecho privado sin fin de lucro lo sea, pero no hay norma que autorice o prohíba al fondo ser emisor. Las consecuencias son relevantes, ya que la Corporación o Fundación puede acceder al Mercado de Valores cumpliendo con su registro en la CMF y como ODP que tiene un objeto exclusivo que se explota a través del FDP, pudiendo entrar al mercado como Corporación o Fundación y después posteriormente acordar aportar al fondo los ingresos recibidos por la emisión de un título de deuda, pero con esta solución el FDP no podría emitir ni recibir directamente los dineros obtenidos. Al contrario, si consideramos que el FDP pueda ser emisor, no necesitaría de la aprobación de una asamblea de la Corporación o de la Fundación para buscar financiamiento, sino solo de la autorización de la Comisión de Deporte Profesional que es el órgano administrador de la misma ${ }^{77}$.

En conclusión, se estima que las corporaciones y fundaciones que desarrollen actividades deportivas profesionales pueden ser emisores de valores de oferta pública si lo desean, en concreto de títulos de deuda, y una vez obtenido el financiamiento aportar los fondos al FDP en virtud del artículo 28 de la Ley $\mathrm{N}^{\circ} 20.019$, de 2005, que regula la constitución del fondo, especialmente las letras e) y f) que aluden a recursos e ingresos que la respectiva corporación o fundación destinen al Fondo ${ }^{78}$.

\footnotetext{
76. Art. 3: "Para los efectos de esta ley, se entenderá por valores cualesquiera títulos transferibles incluyendo acciones, opciones a la compra y venta de acciones, bonos, debentures, cuotas de fondos mutuos, planes de ahorro, efectos de comercio y, en general, todo título de crédito o inversión...."

77. Artículo 30 de la Ley No 20.019: "El Fondo de Deporte Profesional será administrado por una Comisión de Deporte Profesional compuesta por el Presidente de la corporación o fundación, quien la presidirá, y por cuatro miembros o directores. Este Fondo se considerará organismo esencial para los efectos de lo previsto en el artículo 40 de la Ley No 19.712, 2001, del Deporte....”

78. Artículo 28.- El Fondo de Deporte Profesional estará constituido por: a) Las cuotas ordinarias y extraordinarias que la asamblea general acuerde destinar a este objeto; b) Las donaciones que se efectúen a la organización deportiva profesional a cualquier título; c) Los derechos que correspondan a la organización deportiva profesional o que le asignen la federación, asociación, liga $\mathrm{u}$ otras instituciones a que ésta pertenezca; d) Los ingresos provenientes de la comercialización de los espectáculos deportivos profesionales y de los bienes y servicios conexos; e) Otros recursos que anualmente la corporación o fundación destine al Fondo, y f) Todos los demás ingresos que se destinen al Fondo.
} 


\subsection{El gasto y el control de los dineros obtenidos}

Hemos visto y analizado las diversas formas de financiamiento de los clubes deportivos, lo que genera que cada uno de ellos disponga de un presupuesto anual determinado para cumplir sus obligaciones respecto de terceros e invertir en jugadores o en infraestructura deportiva. A nivel interno, la ANFP en el Reglamento de la Asociación Nacional de Fútbol Profesional y en el Reglamento Nacional de Licencia de Clubes contiene normas relacionadas con el denominado Fair Play Financiero, concepto que surge adoptado por la Unión de Federaciones Europeas de Fútbol (UEFA) en el año 2009 con ocasión de los problemas económicos que habían esperimentado clubes como Rangers FC o Valencia CF por los excesivos gastos incurridos y la deficiente administración presentada ${ }^{79}$. Por ello la UEFA aprobó una regulación para solucionar o evitar dichos problemas en el año $2010^{80}$. En el reglamento ANFP se establece un límite de gasto en el artículo 3.5.2.1: "Las partidas de los EERR (estados de resultado) auditados de los clubes correspondientes a remuneraciones de jugadores y cuerpo técnico del primer equipo; otras asignaciones que vayan en beneficio de jugadores y cuerpo técnico; pago por préstamos de jugadores; pago de comisiones de agentes de jugadores, agentes de clubes y/o intermediarios en general; amortizaciones de derechos de jugadores; y, los pagos por la cesión de derechos de imagen de jugadores, no podrá superar el $70 \%$ del total de ingresos generados por el club, considerando el promedio de las ÚLTIMAS tres temporadas móviles." La sanción ante el incumplimiento es la prohibición la prohibición absoluta de inscribir nuevos jugadores en el Registro de Jugadores Habilitados, para el próximo período de inscripciones ${ }^{81}$.

De acuerdo con el Reglamento, para obtener la Licencia de clubes $^{82}$, las ODP deben cumplir con criterios de carácter financiero ${ }^{83}$, siendo los objetivos de ellos: a) Mejorar la capacidad económica y financiera de los clubes; b) Aumentar la transparencia y la credibilidad de los clubes; c) Alentar a los clubes a operar con sus propios ingresos; c) Dar la importancia necesaria a la protección de los acreedores; d) Garantizar el cumplimiento de las deudas y obligaciones laborales con sus jugadores ${ }^{84}$. A su vez,

79. KAPLAN (2015) pp. 709-802.

80. En la actualidad rige la UEFA Club Licensing and Financial Fair Play Regulations en su edición del año 2018 junto con el addendum aprobado el 18 de junio de 2020.

81. Art. 3.5.2.2 Reglamento ANFP.

82. De acuerdo al artículo 1 del Reglamento de Licencias, se entiende por Licencia la certificación que acredita el cumplimiento por el Licenciatario de los requisitos mínimos obligatorios para participar en las competiciones de clubes organizadas por la Confederación y/o la ANFP.

83. Como por ejemplo, presentar un presupuesto anual, contar con estados financieros auditador en cumplimiento con la circulares de la CMF y presentar una declaración jurada ante notario donde se señale que no tienen deudas vencidas con otros clubes, jugadores o entidades. Véase al respecto el art. 21 del Reglamento de Licencias.

84. Art. 21 Reglamento de Licencias. 
se declara que el cumplimiento de ello puede ayudar a salvaguardar la continuidad y la integridad de las competiciones; mejorar la confianza en la probidad de la industria del fútbol profesional; crear un mercado más atractivo para los socios comerciales e inversionistas; y mejorar la capacidad de generar ingresos y la gestión de los gastos ${ }^{85}$. Al contar con mayor información financiera de un club deportivo, un inversionista está en mejores condiciones de tomar la decisión invertir en el mismo, lo que también se hace aplicable a las empresas que deseen celebrar contratos de patrocinio con el equipo.

La Ley de SADP establece una fiscalización y supervigilancia financiera que recae en la CMF, específicamente relativa a los presupuestos, estados financieros, balances y estados de cuentas de las ODP. Además, dicha Ley contiene normas especiales frente al riesgo de insolvencia de que pueden ser objeto las ODP, estableciendo en el caso de las SADP un plazo de 30 días para normalizar la situación, transcurrido el plazo y si no se ha solucionado se establece que debe realizar la convocatoria para un aumento de capital, y si es rechazado el aumento, o si se aprueba pero no se entera dentro del plazo establecido o si la CMF no aprueba los términos de la convocatoria, “...la sociedad no podrá aumentar el monto global de sus colocaciones requerido para restablecer su situación financiera ni podrá efectuar inversiones, cualquiera sea su naturaleza, a menos que se trate de instrumentos emitidos por el Banco Central de Chile $^{86}$. En el caso del FDP, la Comisión del Deporte Profesional debe informar a la CMF del riesgo de insolvencia y se señalarán las medias que se deban tomar a corto plazo para remediar la situación. La misma Ley en el artículo 32 inciso segundo define cuándo se produce el riesgo de insolvencia aplicable al FDP, en concreto, cuando se haya cesado en el pago de una o más obligaciones. Además, se consagran dos situaciones en las cuales se establece presunción de estado de insolvencia notoria: a) $\mathrm{Si}$ transcurridos noventa días desde que se informó a la CMF del cese del pago y no se han solucionado las obligaciones; y b) Si durante un plazo de seis meses se han dejado de cumplir tres o más obligaciones distintas.

\section{Conclusiones}

Durante el presente trabajo se ha podido constatar que los clubes de fútbol profesional chilenos al desarrollar su actividad comercial cuentan con diversas fuentes de financiamiento provenientes ya sea de la normativa deportiva interna como del ordenamiento jurídico en general, como son los premios en dinero por la participación en los torneos; la celebración de contratos de patrocinio o licenciamiento; o de otros actos y operaciones mercantiles que se encuentran disponibles en el ámbito empresarial tradicional.

85. Art. 21 Reglamento de Licencias.

86. Art. 22 Ley SADP. 
La regulación chilena permite a los clubes deportivos celebrar diveros actos y contratos para la búsqueda de mayores ingresos y con ello lograr fines deportivos y/o económicos, pero podemos a la vez identificar algunos problemas relativos al acceso a dicho financiamiento, como es la desigualdad que proviene del carácter de la ODP, ya que el modelo de operación y funcionamiento de una SADP en relación a una Corporación o Fundación que haya constituido un FDP tiene importancia para obtener financiamiento por parte de terceros.

Los derechos de transmisión y su explotación cada vez tienen más relevancia a nivel global y nuestro país no es la excepción al respecto, siendo la fuente de ingresos más importante para los clubes chilenos, pero cuyo modelo actualmente vigente es objeto de cuestionamientos en relación con las normas que atentan a la libre competencia. Si la crítica al modelo resulta o no justificada, no impide señalar que el modelo actual o uno modificado, continuará siendo una de las fuentes de financiamiento más importantes con que cuentan los equipos y el fútbol en general.

Por último, se ha constatado la adopción de diversas medidas financieras, tanto por el legislador, como por la normativa de la ANFP, para controlar el gasto y fiscalizar a clubes fútbol con el objeto de transparentar la información al mercado, garantizar el pago de sus obligaciones y evitar caer en insolvencia.

\section{Referencias bibliográficas}

\section{a) Doctrina}

ASOCIACIÓN NACIONAL DE FÚTBOL PROFESIONAL (2020): Anuario Financiero del Fútbol Chileno Temporada 2019 Tercera edición.

BENTLY, Lionel, SHERMAN, Brad, GANJEE, Dev, \& JOHNSON, Phillip (2018): Intellectual Property Law (OXFORD, Oxford University press, $5^{\mathrm{a}}$ ed. actualizada).

BLACKSHAW, Ian (2012): Sports Marketing Agreements: Legal, Fiscal and Practical Aspects (The Hague, Springer).

BLACKSHAW, Ian (2017): International Sports Law: An Introductory Guide (The Hague, Springer).

CAÑIZARES, Eva (2015): "La venta de los derechos audiovisuales y la compleja aplicación al fútbol del derecho de la competencia". En MILLÁN, Antonio (coord.) Derecho del fútbol: presente y futuro. (Madrid, Editorial Reus), pp. 27-44.

CONFEDERACIÓN SUDAMERICANA DE FÚTBOL (2021): Manual de Clubes CONMEBOL Libertadores.

CORREA, Juan Luis y PINOCHET, Francisco (2016): Regulación jurídica de las transferencias de jugadores de fútbol profesional. Disponible en http://repositorio. uchile.cl/handle/2250/140832 [Fecha de consulta: 20 de enero de 2021]. 
DE LA IGLESIA, Eduardo (2016): Derecho patrimonial y deporte (Madrid, Editorial Reus).

DELOITTE SPORTS BUSINESS GROUP (2020): Football Money League (Manchester).

ECHEBERRY, Sandra (2015): El contrato de patrocinio deportivo (Madrid, Editorial Reus).

FÉRNANDEZ-NÓVOA, Carlos; OTERO, José Manuel; y BOTANA, Manuel (2017): Manual de Propiedad Industrial (Madrid, Marcial Pons, Tercera Edición).

GUERRERO, Antonio (2013): "El movimiento olímpico. Las federaciones deportivas internacionales". En PALOMAR, Alberto. (coord.) Derecho del Deporte. (Navarra, Editorial Aranzadi), pp. 123-176.

Historia de la Ley N. 20.019 (2005): “Crea Sociedades Anónimas Deportivas”. Disponible en: https://www.bcn.cl/historiadelaley/nc/historia-de-la-ley/5577/ [Fecha de consulta: 25 de enero de 2021].

KAPLAN, Valerie (2015): "UEFA financial fairplay regulations and european union antitrust law complications". En Emory International Law Review, Vol. 29, pp. 799857.

RÍOS, Mauricio y ÁLVAREZ, Nicolás (2019): "La Autonomía del Fútbol y su Manifestación en los Métodos de Resolución de Conflictos: El Caso Chileno". En Revista de Derecho, № 246, pp. 105-138.

SCHMITZ, Christián (2012): “Distintividad y uso de las marcas comerciales”. En Re-

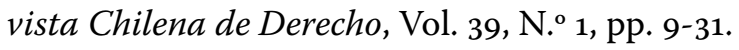

\section{B) Fuentes legales}

Código del Trabajo de Chile.

Ley No 18.045, Ley de Mercado de Valores, 1981.

Ley No 19.039, Establece Normas Aplicables a los Privilegios Industriales y Protección de los Derechos de Propiedad Industrial,1991.

Ley N²0.019, Regula las Sociedades Anónimas Deportivas Profesionales, 2005.

\section{C) Normativa deportiva}

Bases Campeonato Nacional de Primera División Temporada 2021.

Estatutos de la Asociación Nacional de Fútbol Profesional, versión del año 2020.

Estatutos de la Federación de Fútbol de Chile. 
Reglamento sobre el Estatuto y Transferencia de Jugadores de la FIFA, edición de enero de 2021.

\section{D) Documentos}

AZUL AZUL S.A. (2019): Memoria Anual y Estados Financieros, Santiago.

BLANCO Y NEGRO S.A (2019): Memoria Anual, Santiago.

CLUB DE DEPORTES SANTIAGO WANDERERS S.A.D.P (2019): Memoria Anual, Valparaíso.

CRUZADOS S.A.D.P. (2019): Memoria Anual, Santiago.

FISCALÍA NACIONAL ECONÓMICA (2020): Requerimiento en contra de Canal del Fútbol SpA. 